\title{
High-voltage Pulse Generation Using Electrostatic Induction in Capacitor
}

\section{Taku Saiki}

Department of Electrical and Electronic Engineering, Faculty of Engineering Science, Kansai University, Suita, Japan

\section{Email address:}

tsaiki@kansai-u.ac.jp

\section{To cite this article:}

Taku Saiki. High-voltage Pulse Generation Using Electrostatic Induction in Capacitor. International Journal of Electrical Components and Energy Conversion. Vol. 5, No. 2, 2019, pp. 20-29. doi: 10.11648/j.ijecec.20190502.11

Received: October 19, 2019; Accepted: November 18, 2019; Published: November 22, 2019

\begin{abstract}
Pulse power sources can produce high temperature or high-density extreme conditions within a short time. They have been introduced to various fields such as those in laser, fusion research, the production of plasma, shockwaves in water, water treatment, and exhaust gas treatments. Various high voltage pulse sources using insulated-gate bipolar transistor (IGBT) semiconductors with high voltage resistivity have been used. Also, Marx bank circuit is well known as an instrument that produces voltage pulses with low repetitive rates. These instruments have several advantages. However, their problems are a complex structure, high cost, and excessive weight. A simple method of producing high voltage pulses with short rising times based on electrostatic induction in external capacitor used for pulse power applications is proposed. The circuit has a simple structure and contains a minimum number of parts, which makes the instrument small and light weight. In fact, the generation of sawtoothed high voltage pulses with short rising times and low repetitive rates of a few $100 \mathrm{~Hz}$ was successfully conducted in experiments. Theoretical analysis was simultaneously undertaken. The numerically calculated results for generating high voltage pulses were goodly consistent with the experimental ones. Moreover, it has been confirmed that amplification of the output voltage by electro-hydrodynamics (EHD) electricity generation using a jet flame resulted in higher voltage pulses, lower electricity consumption, and high repetition rates.
\end{abstract}

Keywords: High Voltage Pulses, EHD Power Generation, Flame Jet, Electrostatic Induction

\section{Introduction}

Pulse power sources, which can produce high temperature or high-density extreme conditions by releasing electric or magnetic energy stored in coils or capacitors within a short time, have been introduced to various fields such as those in laser [1], fusion research [2], the production of plasma, shockwaves in water, water treatment, and exhaust gas treatments [3-13].

Various pulse power sources have now been developed due to the expanding fields of application [3-19]. The required conditions for pulse power sources differ according to applications. The required parameters for the pulse power sources of individual applications are high voltage, high current, voltage or current pulses with short rise times, large averaged output power, and high repetitive rates.

The generation of pulses using discharge switches typified by a conventional gap switch has been continuously researched [6,19]. Also, various high voltage pulse sources using insulated-gate bipolar transistor (IGBT) semiconductors with high voltage resistivity have been used as alternative switches [15-16]. The Marx bank circuit is well known as an instrument that produces voltage pulses with low repetitive rates [6]. These instruments or electrical circuits have several advantages. However, their three main problems are: 1) a complex structure, 2) high cost, and 3) excessive weight, which is very hard to reduce.

This paper proposes a new method of easily producing sawtoothed high voltage pulses with short rising times and low repetitive rates based on electrostatic induction in the external capacitor.

I adapted electrostatic induction used in an Electrophorus [20] to generate high voltage pulses in this study. Monopole charges are generated in the Electrophorus repeatedly. The repetitive pulse power source using the conventional discharge gap directly supplies electrical power from the power source into the capacitor once. The electrical power is consumed at load. Pulse compression circuits may often be 
added to them. In this method, unlike the conventional one, the load is electrically discontinuous, and substantially less direct current is used for charging from the power supply. A large difference in operation occurs, and the electric energy is repeatedly charged in the capacitor indirectly through electrostatic induction using a high voltage power supply.
The proposed circuit has a simple structure and no moving parts. Thus, the instrument can be small, light weight, and have a long lifetime. In addition, an experiment on high voltage pulses that were amplified due to EHD power generation using a jet flame was also carried out to find whether the electric power of the power source was reduced.

\section{Principle for Generation of High Voltage Pulses}

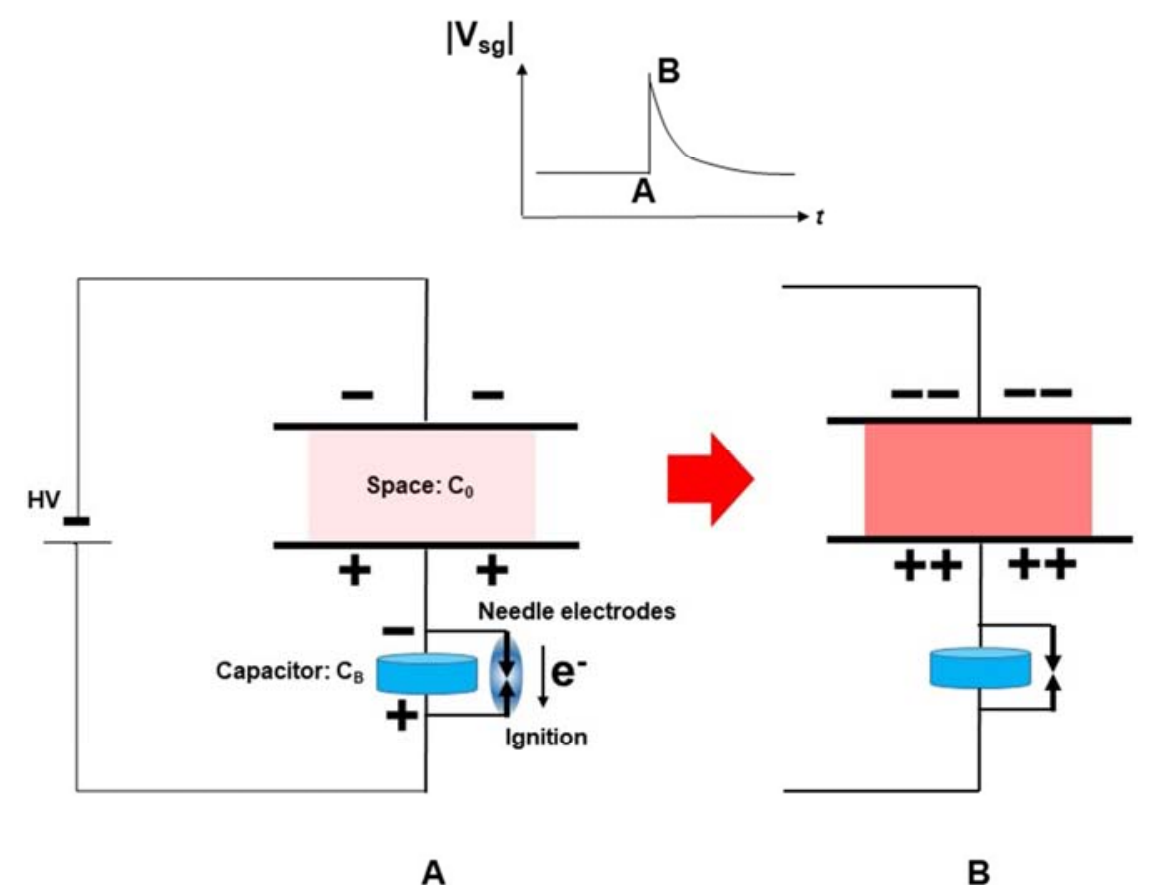

Figure 1. Principle underlying generation of high voltage in space sandwiched by electrodes. $V_{\text {sg: }}$ Voltage of the capacitor that consists of space and the two electrodes.

The principle underlying the generation of high voltage pulses by charging and discharging external capacitors is outlined below. The space between two electrodes can be seen as a capacitor with a capacitance of $C_{0}$ and an external capacitor with a capacitance of $C_{B}$, as shown in Figure 1. Here, $C_{B}$ is much larger than $C_{0}$. We have called the external capacitor a boosting capacitor below. Here, the space between the electrodes is assumed to be filled with gas such as air or low pressure gas. A boosting capacitor is connected in series with the capacitor that consists of the space and electrodes. The capacitors connected in series are connected to a high voltage power supply. The boosting capacitor has needle-needle electrodes. When a high voltage is added to this circuit once, the boosting capacitor is charged to a high voltage due to electrostatic induction by HV supply. The voltage of the boosting capacitor reaches the threshold voltage of the needle-needle electrodes for discharge, which occurs between the electrodes that shorten, and negative charge flows out of the boosting capacitor (Figure 1A). It means that positive charge flows to the below electrode of the capacitor which consists of space and two electrodes. A large current flows between electrodes within a short time. If the discharge has negligible energy loss, all the stored electrical energy in the boosting capacitor migrates to the capacitor, which consists of space and two electrodes. A very high voltage is generated in the capacitor, which consists of space and the two electrodes (Figure 1B). This is because the series connected capacitors are recognized as a single capacitor with variable capacitance. The boosting capacitor is ignored by the shortening of the needle-needle electrodes when discharge occurs.

After discharging in needle-needle electrodes, the moved and stored positive charges at the below electrode of the capacitor consisting of a space and two electrodes remain because the electrical connection is cut.

The boosting capacitor re-charges using the electrostatic induction in the boosting capacitor. I call the device a boosting capacitor because, when charged, it releases the electrical power repeatedly and enhances the density of the positive charges in the lower electrode of the space gap. Also, the boosting capacitor can be considered a pulse current source in principle.

Obviously, the principle of charging in the capacitor and generation of high voltage in a capacitor consisting of a space and two electrodes is similar to one of an Electrophorus [20]. The discharges in needle-needle electrodes have almost the same effect as touching the Electrophorus with one's fingers. When positive and negative charges are stored in the Electrophorus, the charges on the upper surface are removed after fingers touch it. After that, the Electrophorus moves away from the dielectric body while keeping the charges, and high voltage occurs because 
the effective capacitance decreases.

The stored electrical energy of the capacitor that consists of space and the two electrodes is explained next.

$$
E_{0}=\frac{1}{2} \cdot C_{0} \cdot V_{0}^{2}
$$

Here, $V_{0}$ is the voltage that is added to the capacitor that consists of space and the two electrodes.

Also, the equation for the stored electrical energy of the boosting capacitor is:

$$
E_{B}=\frac{1}{2} \cdot C_{B} \cdot V_{B}^{2}
$$

Here, $V_{B}$ is the voltage to the boosting capacitor when discharge occurs. After discharge has occurred, all the stored electrical energy in the boosting capacitor migrates to the capacitor that consists of space and the two electrodes

The new electrical energy of the capacitor that consists of space and the two electrodes is next changed as:

$$
E_{0}{ }^{\prime}=E_{0}+E_{B}
$$

Thus, the equation for $V_{0}^{\prime}$ after discharge is:

$$
V_{0}^{\prime}=\sqrt{\frac{2 \cdot E_{0}^{\prime}}{C_{0}}}
$$

The stored charge in the boosting capacitor by the move of discharge to the capacitor consists of space and the two electrodes. After that, the moved charges slowly disappear. The equation for the increment of the stored charge at the positive electrode is:

$$
\Delta Q_{0}=Q_{0}^{\prime}-Q_{0}=C_{0} \cdot V_{0}^{\prime}-C_{0} \cdot V_{0}=C_{0} \cdot\left\{\sqrt{\left(\frac{C_{B}}{C_{0}}\right) \cdot V_{B}^{2}+V_{0}^{2}}-V_{0}\right\}
$$

Here, $Q_{0}>0$. This $\Delta Q_{0}$ is newly stored in the capacitor that consists of space and the two electrodes for each discharge. $\Delta Q_{0}\left(t_{0}\right)$ is the supplied charge at discharge, and $Q_{0}$ is calculated by:

$$
\frac{d Q_{0}(t)}{d t}=+\Delta Q_{0} \cdot \delta\left(t_{0}\right)-I(t)
$$

where $t_{0}$ is when discharge occurs. The equation for the current that flows out of the capacitor is:

$$
I(t)=\frac{V(t)}{R_{\mathrm{p}}}=C_{0} \cdot \frac{d V(t)}{d t}
$$

where $R_{p}$ is the internal resistance of the capacitor. The equations from (1) to (8) were converted to differential equations, and the charge, voltage, and currents of the capacitor were calculated.

The equation for the repetition time is:

$$
\tau \propto \frac{C_{B} \cdot V_{B} \cdot R_{S}}{V_{O C W}}
$$

Here, $R_{S}$ is the charging resistance, and $V_{O C W}$ is the output voltage of the rectification circuit. The time depends on $C_{B}, V_{B}$, $R_{s}$, and $V_{\text {ocw }}$.

\section{Method for Experiment}

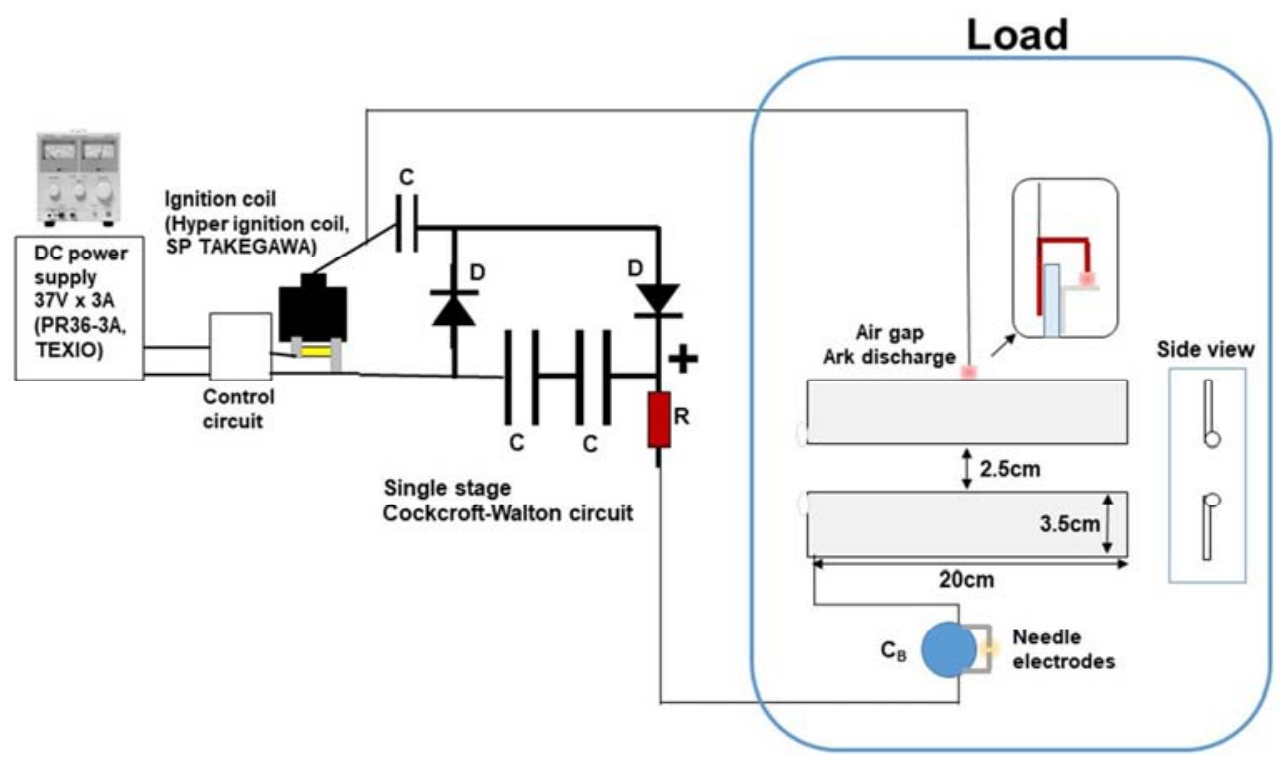

Figure 2. Experimental setup for generating high voltage pulses.

$C_{B}$ : boosting capacitor, D: high voltage withstanding diode

$\mathrm{C}$ : capacitor for rectification, and $\mathrm{R}$ : resistance for measuring current. 
The experimental setup for generating high voltage pulses is outlined in Figure 2. Two capacitors (capacitance of $100 \mathrm{pF}$ (TDK, Japan), withstanding voltage of $3 \mathrm{kV}$, distance of $4 \mathrm{~mm}$ between needle electrodes, capacitance of $1000 \mathrm{pF}$ (Murata Manufacturing, Japan), withstanding voltage of $16 \mathrm{kV}$, and a distance of $2 \mathrm{~mm}$ between needle electrodes) were used in the experiment as the boosting capacitors. Two diodes (Fuji Denki Systems, Japan, with a withstanding voltage of $12 \mathrm{kV}$ ) connected in series were used. A DC power source $(36 \mathrm{~V} \times 3 \mathrm{~A}$, PR36-3A, TEXIO, Japan) was also used. A high voltage generator (a harmonic power source using an ignition coil with a frequency of 20 $\mathrm{kHz}$ and a maximum output voltage of $5 \mathrm{kV}$ ) was connected to the DC power source. The high voltage generator was connected to a single-stage Cockcroft-Walton (CW) circuit (a double voltage rectifier circuit), as outlined in Figure 1. The output was chosen on the plus electrode side of the double voltage rectifier circuit. High voltage enamel resistance with a length of $4.8 \mathrm{~cm}$ and resistance of $18 \mathrm{kohm}$ was connected to the plus output side of the rectifier circuit to monitor the currents.

The load consisted of two aluminum plates (the capacitor consisted of space and the two electrodes. The thickness of both Al plates was $0.5 \mathrm{~mm}$ was used, as indicated on the right of Figure 2. The two aluminum plates were placed vertically. The size of both Al plates were $3.5 \times 20 \mathrm{~cm}$. Their edges were rounded with a diameter of $3 \mathrm{~mm}$. The top of the Al plate had a needle electrode with a $1 \mathrm{~mm}$ gap to the $\mathrm{Al}$ plate. Here, an arc discharge at the needle electrode by increasing the output voltage of the rectifier circuit to enhance the output voltage was generated. The arc discharge has less of a relationship to the characteristic of pulse voltage generation, and we can ignore it as if it is a conduction line.

Here, the circuit point, at which the output of the high voltage power generator and the input of the rectifier circuit were connected, as shown in Figure 2, was connected to the load as an output. The bottom Al plate was connected to the positive output of the rectifier circuit via the boosting capacitor.

A high voltage probe (HV-60, Sanwa, Japan), an oscilloscope (TDS-2012, Textronics, Japan), and a tester (CD731a, Sanwa, Japan) were used for measuring the voltage waveform and the average voltage was added to the load, current. The waveform of the output voltage was pulse when the load was not connected because AC and DC voltage were mixed. When the load and the boosting capacitor were connected to the rectifier circuit, arc discharge occurred at the needle gap and the load current increased, which resulted in degrading the output voltage of the DC power source and the occurrence of relaxed self-excited oscillation.

\section{Results and Discussion}

An experiment to generate high voltage pulses was conducted by using the instrument outlined in Figure 2. The results are provided in Figures 3 and 4

The measured voltage added to the load and current are given in Figure 3 (a). The output voltage of the rectifier circuit was proportionally changed to the output voltage or current of the DC power source when no arc discharge was generated at the needle gap. A maximum average output voltage of $12.5 \mathrm{~kW}$ was obtained for an output voltage of 20 $\mathrm{V}$ and an output current of $0.5 \mathrm{~A}$. The load current increased and the output voltage of DC power supply degraded when arc discharge at the needle gap was generated and $C_{B}=100$ $\mathrm{pF}$ was used. Average output voltages of $12 \mathrm{kV}$ and $19 \mathrm{kV}$ were obtained for the output currents of 1.2 and 2.0 A, as shown in Figure 3 (a). An output peak voltage of around 26 $\mathrm{kV}$ was obtained.

The load current increased, and the output voltage of the DC power supply degraded when arc discharge was generated at the needle gap and $C_{B}=1000 \mathrm{pF}$ was used. Average output voltages of 13 and $18 \mathrm{kV}$ were obtained for the output currents of 1.2 and $2.0 \mathrm{~A}$, as shown in Figure 3 (a). An output peak voltage of around $25 \mathrm{kV}$ was obtained. The repetition rates obtained from the oscilloscope are plotted in Figures 3 ( $a$ and $b$ ).

The repetition rate changed from 80 and to $290 \mathrm{~Hz}$ for the output currents of 1.2 and $2.0 \mathrm{~A}$ when $C_{B}=100 \mathrm{pF}$ was used, as shown in Figure $3(\mathrm{~b})$, whereas the repetition rate changed from 10 and to $40 \mathrm{~Hz}$ for the output currents of 1.2 and $2.0 \mathrm{~A}$ when $C_{B}=1000 \mathrm{pF}$ was used, as shown in Figure 3 (b), due to the increasing charge time of the boosting capacitor.

The waveforms of applied voltage to the load obtained by oscilloscope at $C_{B}=100 \mathrm{pF}$ and without arc discharge are given in Figure 5, where the $20 \mathrm{kHz} A C$ component appeared in the waveform. Sawtoothed high voltage pulses applied to the load with the repetition rate of $7.1 \mathrm{~Hz}$ were observed. The voltage increments applied to the load within a short time were $6 \mathrm{kV}$ when discharge occurred at the needle electrodes of the boosting capacitor. After that, the voltages applied to the load slowly decreased due to outflowing stored charges.

The waveforms of applied voltage to the load obtained by oscilloscope at $C_{B}=100 \mathrm{pF}$ and with arc discharge are provided in Figure 6. Also, sawtoothed high voltage pulses applied to the load with a repetition rate of $290 \mathrm{~Hz}$ were observed, where the output current increased to $1.8 \mathrm{~A}$ and blocking oscillation was observed after arc discharge was generated. The voltage increment applied to the load within a short time was $4 \mathrm{kV}$ when discharge occurred at the needle electrodes of the boosting capacitor.

The waveforms of the applied voltage to the load obtained by oscilloscope at $C_{B}=1000 \mathrm{pF}$ and with arc discharge are given in Figure 7. Also, sawtoothed high voltage pulses applied to the load with a repetition rate of $33 \mathrm{~Hz}$ were observed, where the output current increased to 2.0 A and blocking oscillation was also observed after arc discharge was generated. The voltage increment applied to the load within a short time was $5 \mathrm{kV}$ when discharge occurred at the needle electrodes of the boosting capacitor. Variations occurred in the voltage applied to the load by 
changing the space gap of the load. However, there was less change in the average or peak voltage applied to the load and the repetitive rate was observed in an experiment.

The waveform of the rise voltage applied to the load at $C_{B}=100 \mathrm{pF}$ is shown in Figure 8. It was found that the rise time was a few tens of nanoseconds. The waveform then indicated an oscillation due to the inductance component. Here, the increment of voltage was $6 \mathrm{kV}$. The waveform of the rise voltage applied to the load observed by using a spherical electrode gap at $C_{B}=1000 \mathrm{pF}$ is given in Figure 9. We found that the rise time was the same, and the waveform indicated an oscillation due to the inductance component. Here, the increment of voltage was $15 \mathrm{kV}$.

The waveform of current at $C_{B}=100 \mathrm{pF}$ is indicated in Figure 10, as an example. This was because the waveform of the output voltage was a pulse, and the current flowed asymmetrically. A larger current flowed when the voltage was high. A pulse current simultaneously flowed in the load at the discharge of the needle electrode gap. The current was evaluated to be $22 \mathrm{~mA}$, which was determined from the voltage of $400 \mathrm{~V}$ at the waveform, as shown in Figure 9. The duration of the discharge pulse was evaluated to be $3.3 \mu \mathrm{s}$.

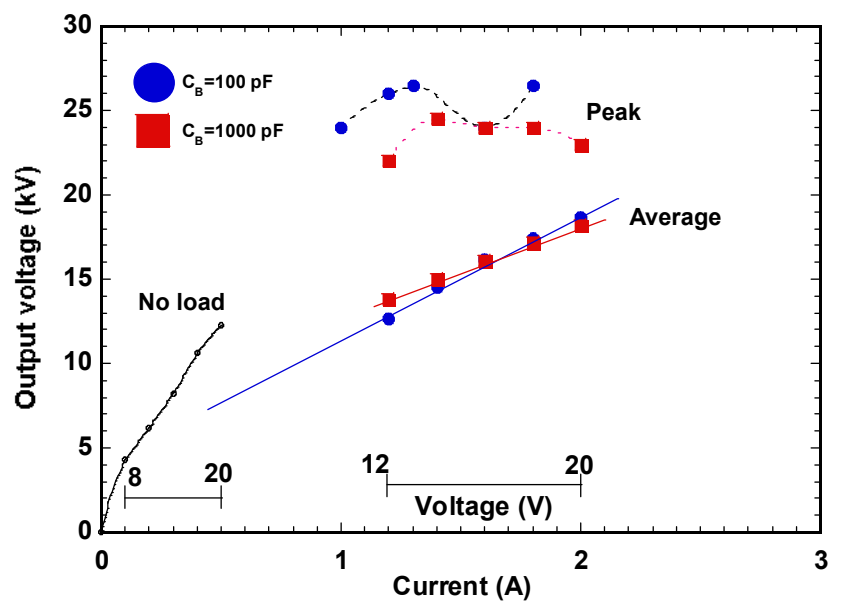

(a)

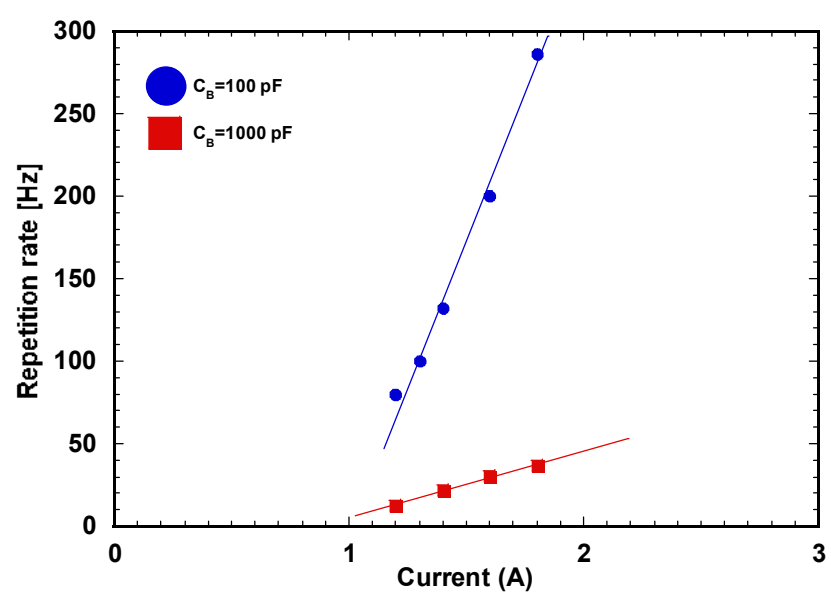

(b)

Figure 3. Properties of added voltage and current applied to load. (a) Average and peak voltage applied to load and (b) repetition rate.

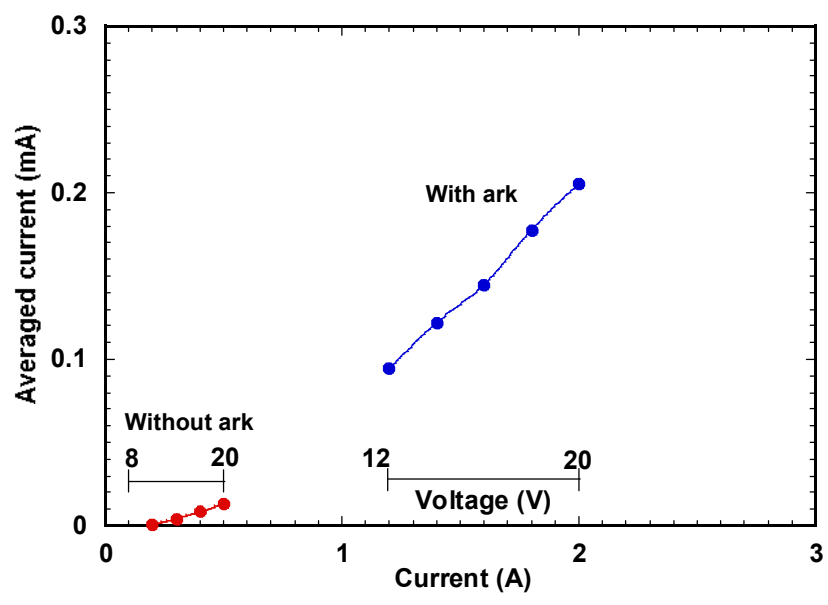

Figure 4. Measured average output current for rectification circuit. Source current was changed from 1.2 to $2.0 \mathrm{~A}$ for $C_{B}=100 \mathrm{pF}$, and average output current for rectification circuit was changed from 0.1 to $0.21 \mathrm{~mA}$. Average output current increased by 20 times compared to when there was no load.

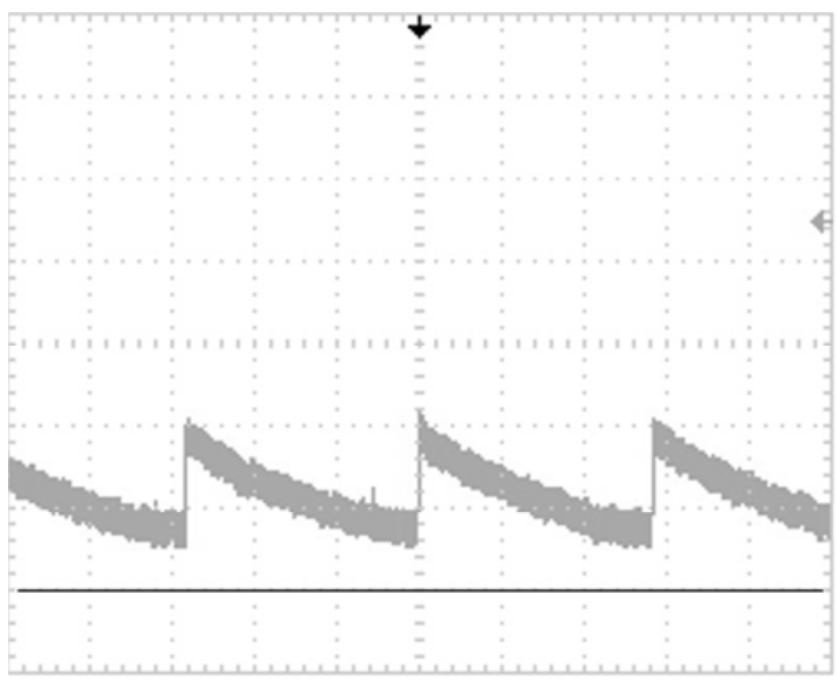

Figure 5. Waveforms of applied voltage to load. Without arc discharge, current: $0.4 \mathrm{~A}$, repetition rate: $7.1 \mathrm{~Hz}, C_{B}=100 \mathrm{pF}, 5 \mathrm{kV} /$ div., and $50 \mathrm{~ms} / \mathrm{div}$.

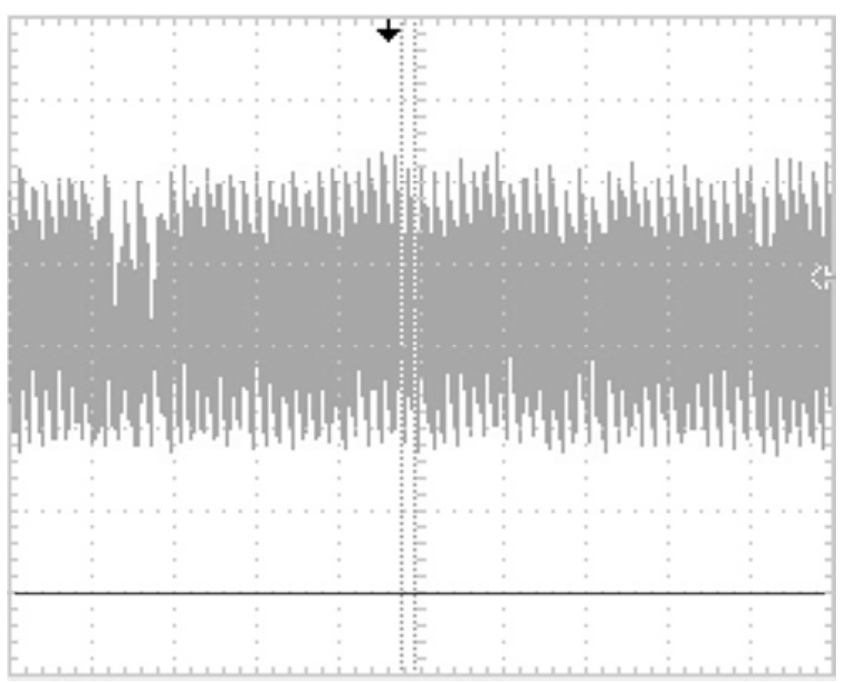

Figure 6. Waveforms of applied voltage to load. Current: 1.8 A, repetition rate: $290 \mathrm{~Hz}, 5 \mathrm{kV} / \mathrm{div}$, and $50 \mathrm{~ms} / \mathrm{div}$. 


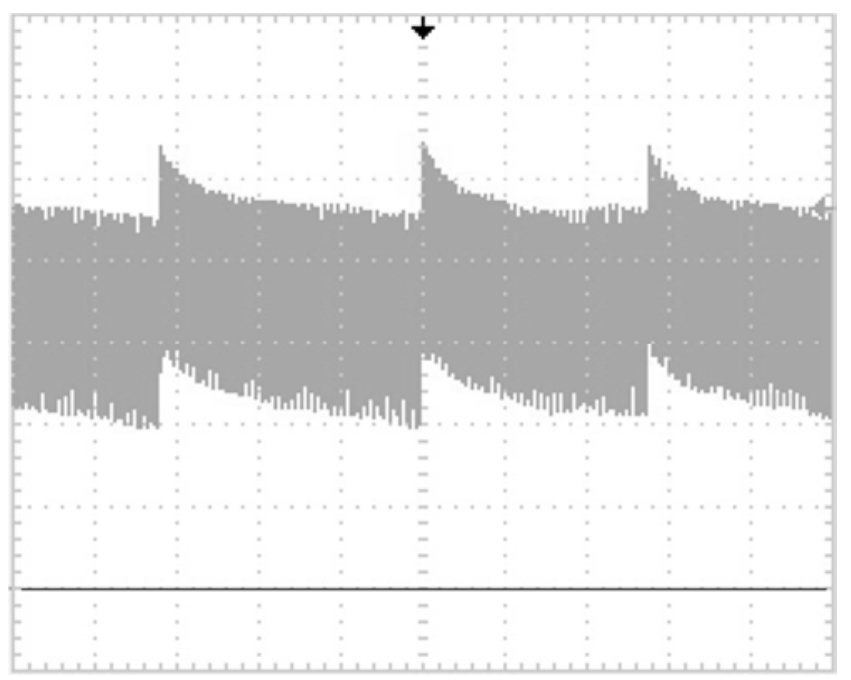

Figure 7. Waveforms of applied voltage to load. Current: 2.0 A, repetition rate: $33 \mathrm{~Hz}, 5 \mathrm{kV} / \mathrm{div}$, and $10 \mathrm{~ms} / \mathrm{div}$.

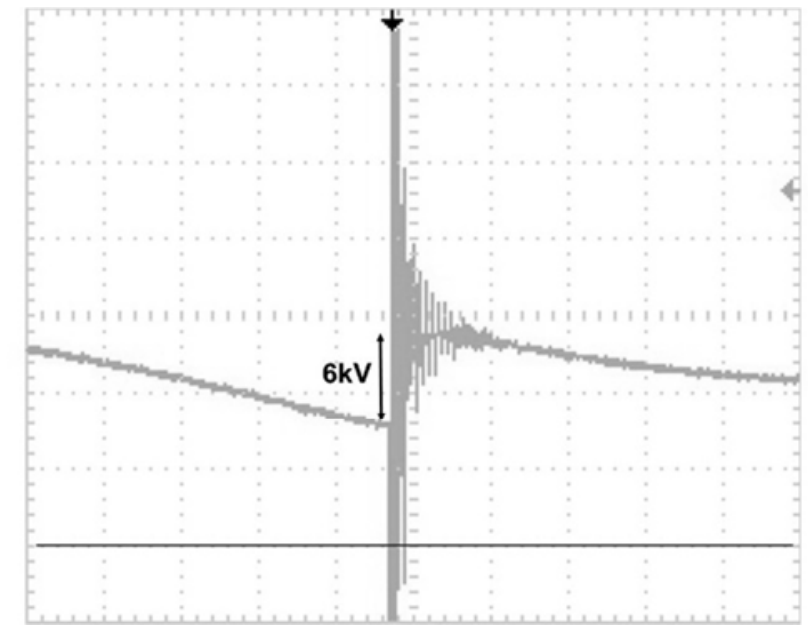

Figure 8. Waveform of applied voltage to load: $C B=100 \mathrm{pF}, 5 \mathrm{kV} / \mathrm{div}$, and 1 us /div.

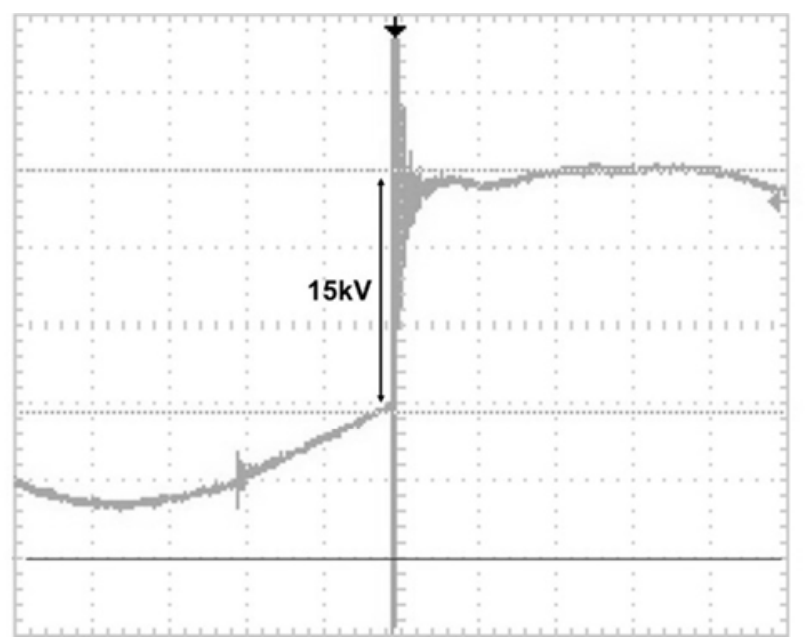

Figure 9. Waveform of applied voltage to load: as an example, $1000 \mathrm{pF}$, sphere gap electrode: $2 \mathrm{~mm}$ gap (discharge voltage: $6 \mathrm{kV}$ ), $5 \mathrm{kV} /$ div, and 2.5 $\mu \mathrm{s} / \mathrm{div}$.

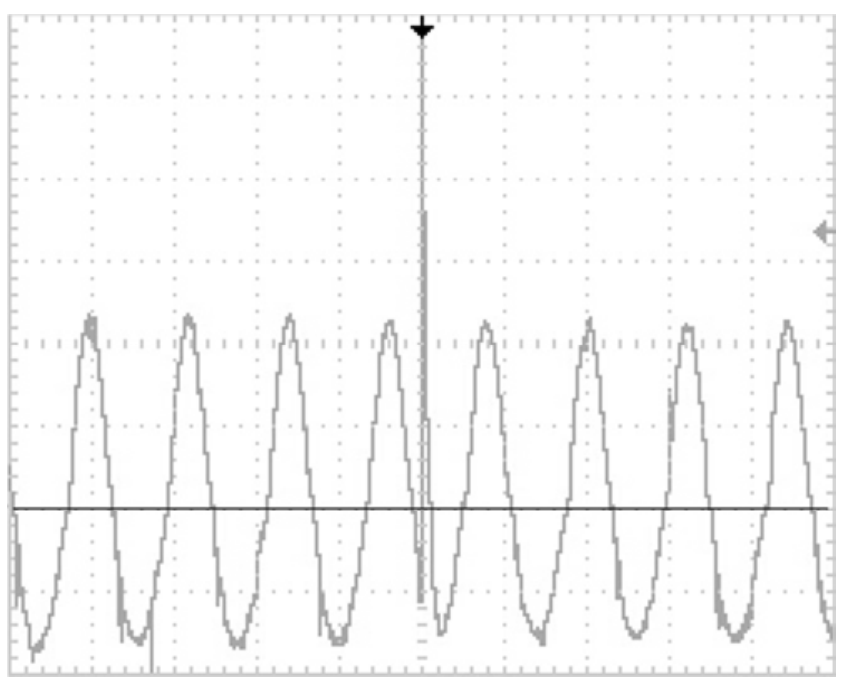

Figure 10. Waveform of current: as an example, output current of DC power source: $1.2 \mathrm{~A}, 5 \mathrm{kV} / \mathrm{div}$, and $25 \mu \mathrm{s} / \mathrm{div}$.

The numerically calculated waveform of the voltage applied to the load, as an example, is shown in Figure 11. Equations (5) to (7) were used for calculating the voltage applied to the load and current and charge, where the repetition rate is $100 \mathrm{~Hz}$ for the calculation parameter. The $V_{B}$ is $4 \mathrm{kV}, C_{0}$ is $10 \mathrm{pF}, C_{B}$ is $100 \mathrm{pF}, V_{i n i}$ is $10 \mathrm{kV}$, and $R_{p}$ is 200 Mohm. The time mesh was set to $10 \mu \mathrm{s}$. The calculated results in Figure 11 are the sawtooth waveforms and are consistent with the results in Figures 5-10. It was found that the output currents that flowed out of the load were $6.5 \mathrm{~mA}$ at peak and there was a weak current of below $0.1 \mathrm{~mA}$ at the charging boosting capacitor.

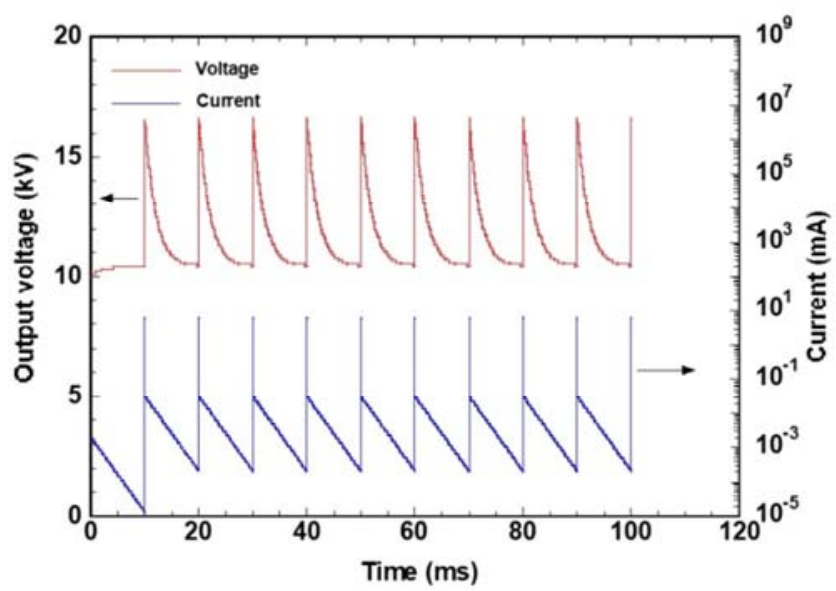

Figure 11. Numerically calculated waveform of voltage applied to load, as an example.

The calculated and measured raised voltage of the pulse during discharge for $V_{B}$ is shown in Figure 12. Equations (1) to (4) were used for calculating the voltage applied to the load. The solid line plots the calculated voltage applied to the load, and the dashed line indicates the calculated raised voltage of the pulse during discharge. Also, $\eta$ in Figure 12 (b) is the energy transfer efficiency from the boosting capacitor to the load. The experimental results are goodly consistent with the numerically calculated ones at $C_{B}=100 \mathrm{pF}$, as 
shown in Figure 12 (a). However, the experimental results are not consistent with the numerically calculated ones at $C_{B}=1000 \mathrm{pF}$, as shown in Figure 12 (b). The measured $V_{B}$ reduced from the calculated ones at $\eta=1.0$. Because the experimental results between using the needle electrode gap and using the spherical electrode gap were almost the same, we expected this to be due to a reduction in capacitance in the ceramic capacitor at high voltage because of material properties. The measured $V_{B}$ was close to the calculated one at $\eta=0.5$. It was found that the stored electrical energy of $0.8 \mathrm{~mJ}$ completely migrated to the load at $C_{B}=100 \mathrm{pF}$.

The raised voltage of the pulse during discharge for $C_{B}$ are plotted in Figure 13. Equations (1) to (4) were also used for calculating the voltage applied to the load. It was recognized that the charges in the load depended on the initial voltage, $V_{i n i}$, that was applied to the load. If $C_{0}=10 \mathrm{pF}, C_{B}=100 \mathrm{pF}, C_{B}=4 \mathrm{kV}$, and $V_{\text {ini }}$ is $0 \mathrm{kV}$, the boosting capacitor has $32 \%$ of the charge stored in the load. If $V_{\text {ini }}$ is $10 \mathrm{kV}$, only $15 \%$ of the charge is stored in the load. The DC component of the output voltage for the rectification circuit must remain low with increasing repetition rate.

The numerically calculated results for the raised voltage of the pulse during discharge are plotted in Figure 14, where $C_{B}=2.5 \mathrm{kV}$ and $V_{\text {ini }}=15 \mathrm{kV}$. When capacitance increased, the raised voltage of the pulse became saturated. It has been recognized that we needed to use a capacitor with lower capacitance and high withstand voltage instead of using a capacitor with high capacitance.

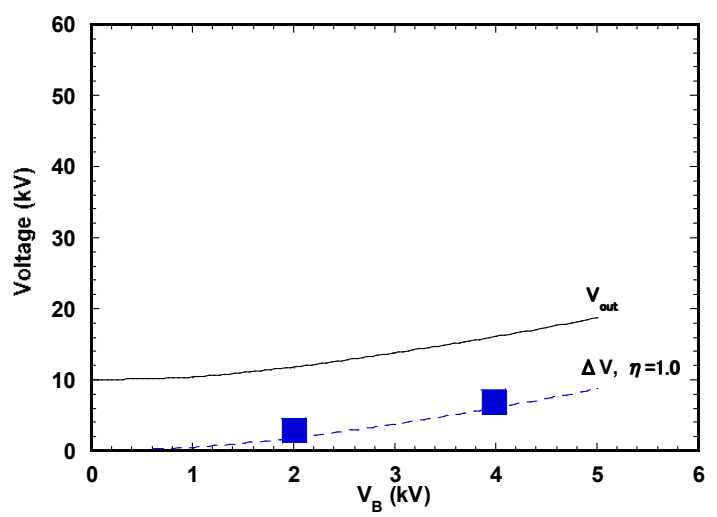

(a)

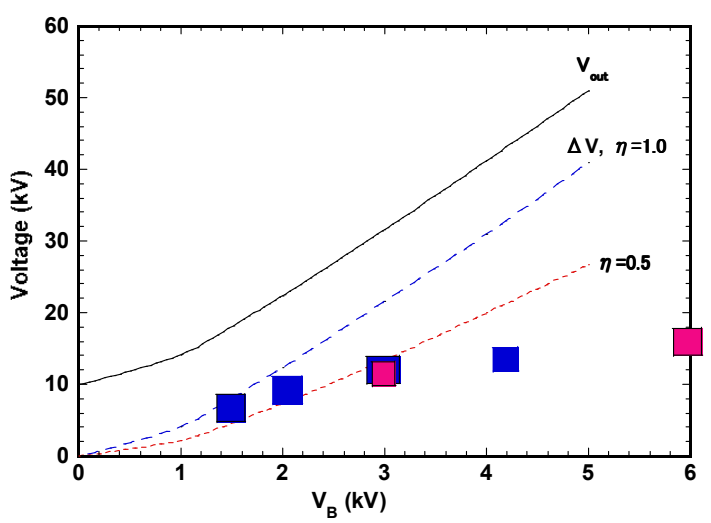

(b)

Figure 12. Voltage applied to load and raised voltage of pulse during discharge for $V B$. (a) $C B=100 \mathrm{pF}$ and (b) $C B=1000 \mathrm{pF}$. Red squares plot experimental results when spherical electrode gap was used, and blue squares plot experimental results when needle electrode gap was used.

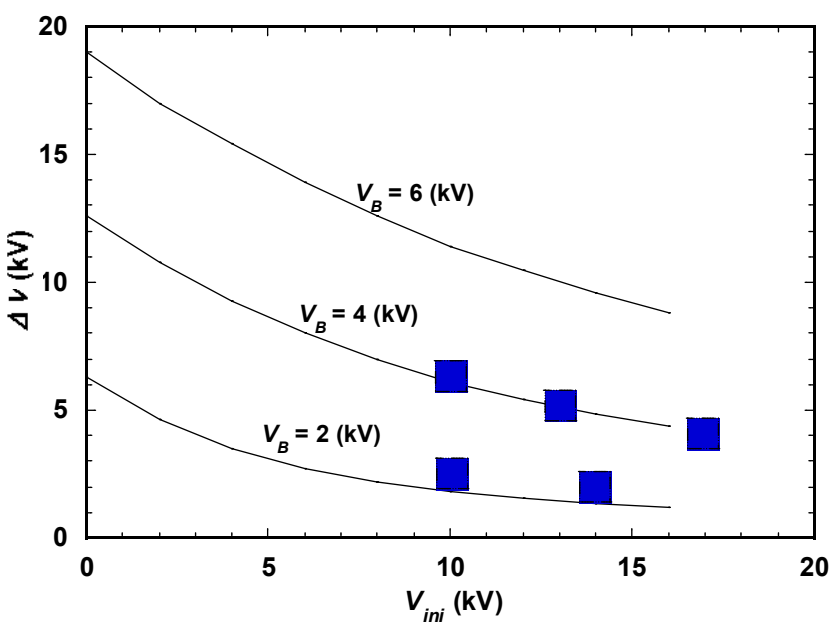

Figure 13. Raised voltage of pulse during discharge for $C_{B} . C_{B}=100 \mathrm{pF}$ and $C_{0}=10 p F$. Solid line plots calculated applied voltage to load. Blue squares plot experimental results when using needle electrode gap.

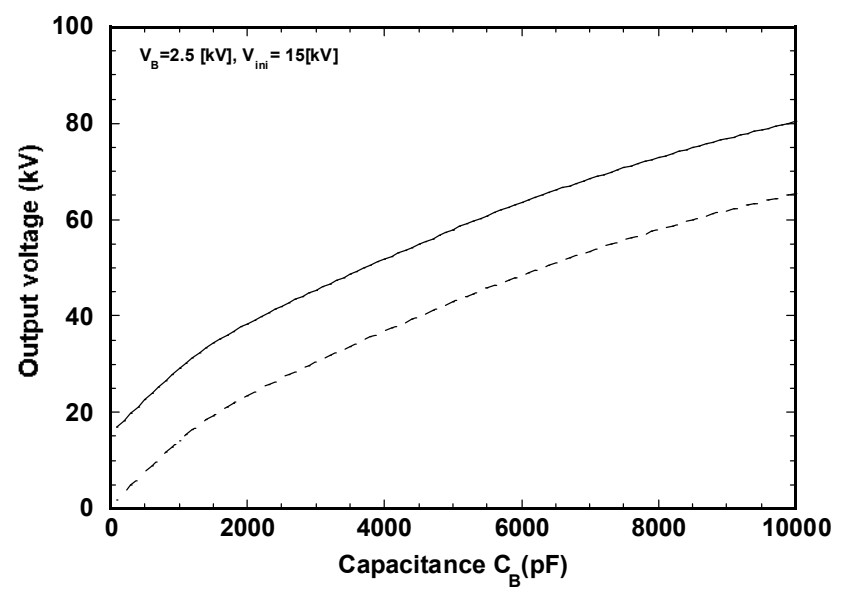

Figure 14. Applied voltage to load and raised voltage of pulse during discharge as function of $C_{B}$. Solid line plots applied voltage to load. Dashed line plots raised voltage of pulse during discharge.

Flame jet EHD power generation [21] was used to enhance the output voltage and reduce consumption power. The structure of the load connected to a single-stage Cockcroft-Walton $(\mathrm{CW})$ circuit was modified to the single Al electrode in Figure 15 to adapt the experiment using flame jet EHD power generation. The EHD power generator uses the following technique. Di-electric fluid or weakly ionized plasma is used for an alternative in the belt to move the electrons. The fluid absorbs some of them. The electrons are accelerated in the fluid. As a result, an effective new high voltage power supply is created, and additional connects are enabled except for the high voltage power source. The negative charges are collected at the front of the flame. A high voltage is generated between the front of the flame and the positive electrode. A negative needle electrode was used for the load, and the flame coming out from a butane burner (flame length: $5 \mathrm{~cm}$ ) was closed, as shown in Figure 15. The direction of the needle electrode is parallel to the bottom Al plate electrode. 


\section{To HV circuit}

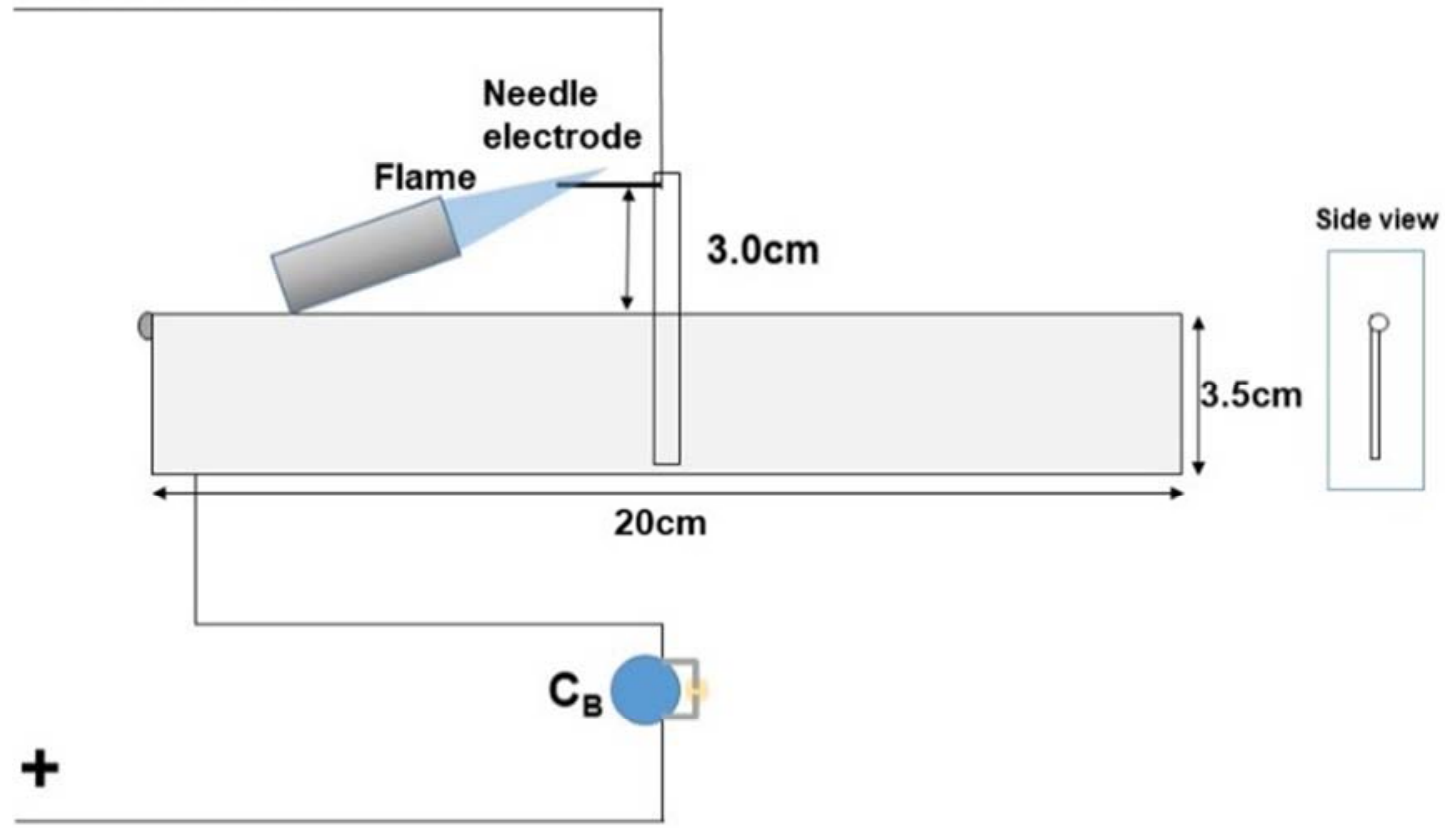

Figure 15. Load using flame jet EHD power generation.

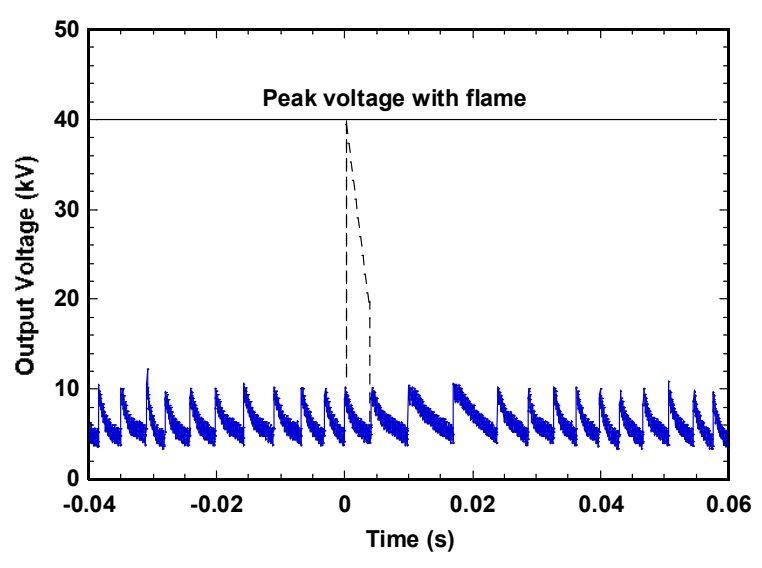

(a)

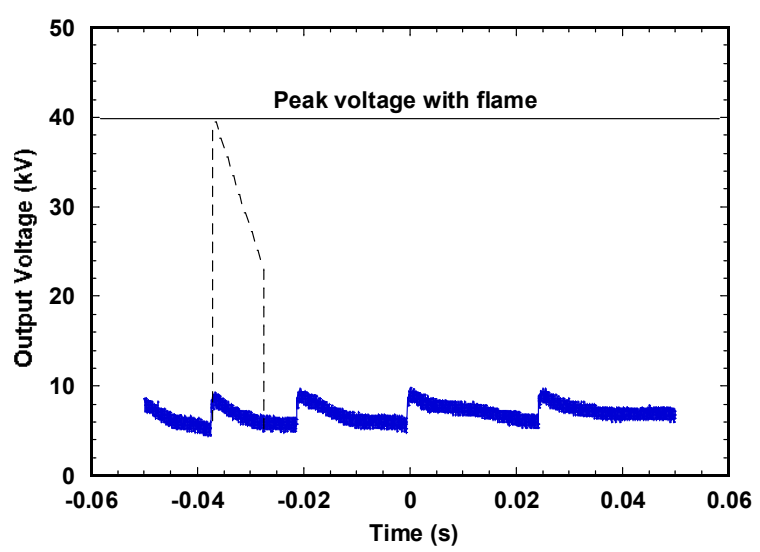

(b)

Figure 16. Applied voltage to load. (a) $C_{B}=100 \mathrm{pF}$, current of power source: $0.5 \mathrm{~A}$, and repetition rate: $130 \mathrm{~Hz}$, (b) $C_{B}=1000 \mathrm{pF}$, current of power source: $0.5 \mathrm{~A}$, and repetition rate: $250 \mathrm{~Hz}$.

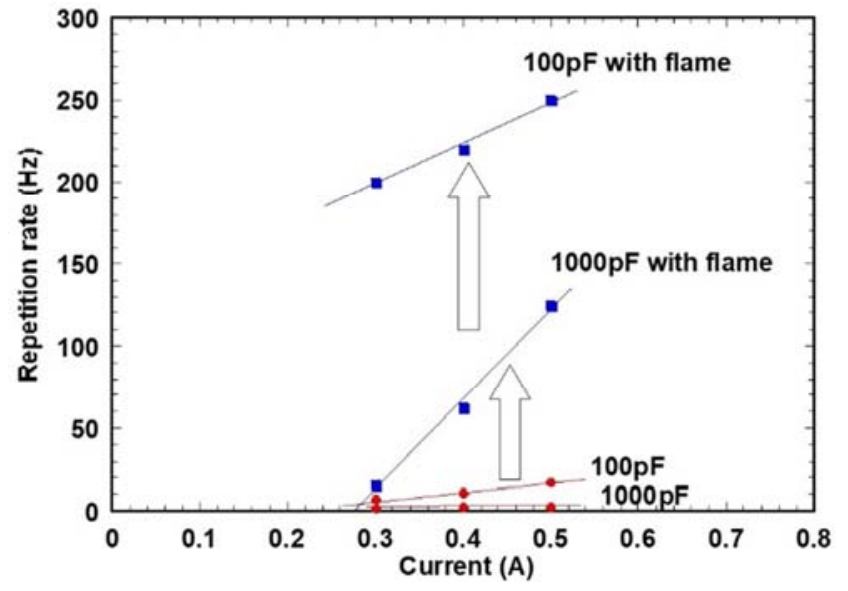

(a)

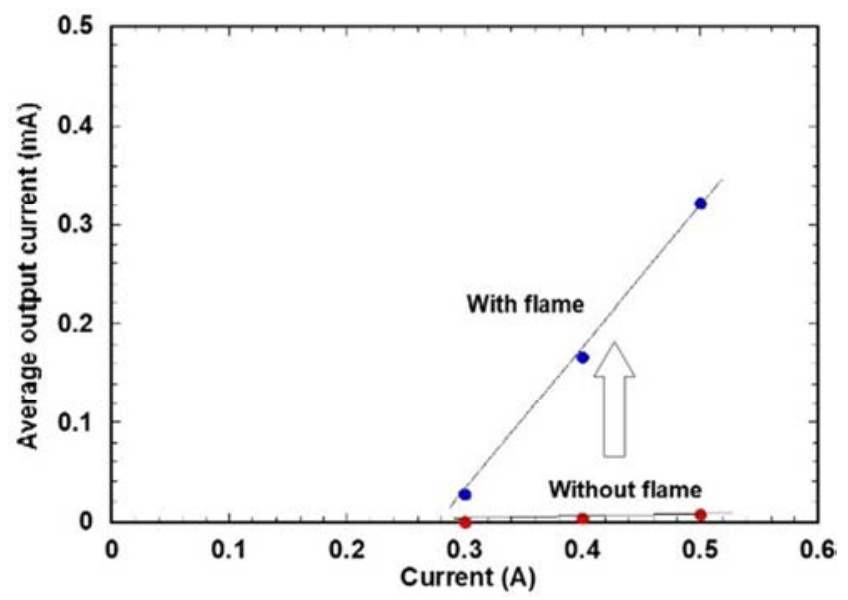

(b) 


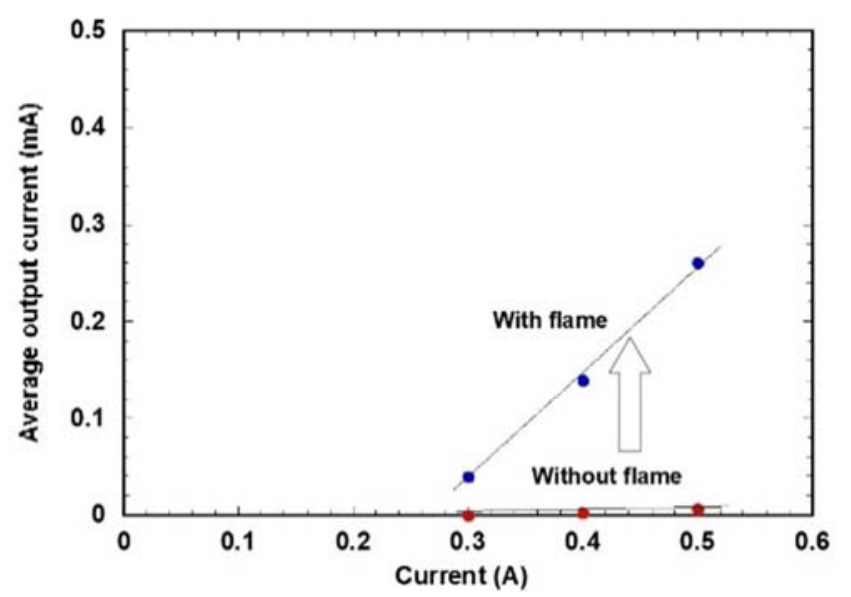

(c)

Figure 17. Properties of circuit. (a) repetition rate, (b) average current: $C_{B}$ $=100 \mathrm{pF}$, and (c) average current: $C_{B}=1000 \mathrm{pF}$.

The distance between the needle electrode and $\mathrm{Al}$ electrode was set to $3.0 \mathrm{~cm}$. When $C_{B}=100 \mathrm{pF}$, the repetition rate was remarkably increased from $17 \mathrm{~Hz}$ to $250 \mathrm{~Hz}$ and a corona discharge from the Al plate electrode was observed. The generated voltage between the flame and the Al plate electrode of the load was estimated to be $40 \mathrm{kV}$, which was estimated from the corona discharge length of $1.5 \mathrm{~cm}$. A maximum amplified voltage of $40 \mathrm{kV}$ was also prospected from the prevous experiment [20].

The measured voltage applied between electrodes is plotted in Figures 16 (a) and (b). The output current of the DC power source was $0.5 \mathrm{~A}$ at $C_{B}=100 \mathrm{pF}$, and the repetition rate increased to $250 \mathrm{~Hz}$. The average voltage between electrodes was $7 \mathrm{kV}$. The output current of the DC power source was 0.5 A at $C_{B}=1000 \mathrm{pF}$, and the repetition rate increased to $130 \mathrm{~Hz}$. The average voltage between electrodes was $6 \mathrm{kV}$.

The repetition rate for the output current of the DC power source is plotted in Figure 17 (a). The repetition rate at $C_{B}=100$ $\mathrm{pF}$ changed to a few $\mathrm{Hz}$ and $20 \mathrm{~Hz}$ for each output current of 0.3 and $0.5 \mathrm{~A}$ when the flame jet was not used. The repetition rate increased to 200 and $250 \mathrm{~Hz}$ for each output current of 0.3 and $0.5 \mathrm{~A}$ when the flame jet was used. The repetition rate at $C_{B}$ $=1000 \mathrm{pF}$ changed to 1.4 and $2.6 \mathrm{~Hz}$ for each output current of 0.3 and $0.5 \mathrm{~A}$ when the flame jet was used. The repetition rate also increased to 16 and $130 \mathrm{~Hz}$ for each output current of 0.3 and $0.5 \mathrm{~A}$ when the flame jet was used.

The measured average output current for the rectification circuit is shown in Figures 17 (b) and (c). The output current of the DC power source at $C_{B}=100 \mathrm{pF}$ is $0.5 \mathrm{~A}$, and the average output current increased to $0.32 \mathrm{~mA}$. The output current of the DC power source is $0.5 \mathrm{~A}$ at $C_{B}=1000 \mathrm{pF}$, and the average output current increased to $0.28 \mathrm{~mA}$.

The results obtained from the experiments are summarized in Table 1.

Table 1. Comparisons of high voltage pulses being generated using relaxed self-excited oscillation and that using flame jet EHD power generation.

\begin{tabular}{llll}
\hline & Output power of supply (W) & Total output power applied to load (W) & Power from boosting capacitor to load (W) \\
\hline $\begin{array}{l}\text { Using only relaxed } \\
\text { self-excited oscillation }\end{array}$ & 40 & 4 & 0.2 \\
$\begin{array}{l}\text { Using flame jet EHD power } \\
\text { generation }\end{array}$ & 10 & (only rectification circuit) 9 (with flame) & 5 (0.3 only rectification circuit) \\
\hline
\end{tabular}

This method easily produced high voltage pulses from a DC power source. The limit of the repetition rate of the voltage pulse should be $1 \mathrm{kHz}$ due to the use of the discharge gap. Part of the output electrical power for the rectification circuit migrated to the boosting capacitor, and the stored electrical energy was injected to the load with a different timing.

The average voltage added to the load was $7 \mathrm{kV}$ when flame jet EHD power generation was used and the average current was $0.32 \mathrm{~mA}$, where the consumption power was evaluated to be $2.1 \mathrm{~W}$. The peak voltage of the load due to voltage amplification of the flame jet EHD increased to around $40 \mathrm{kV}$. This total consumption power at load was evaluated to be $9 \mathrm{~W}$. When the $C_{B}$ was $100 \mathrm{pF}$, the stored electrical energy was $0.8 \mathrm{~mJ}$, the repetition rate was $250 \mathrm{~Hz}$, and the transfer electrical energy from the boosting capacitor to the load was evaluated to be $0.2 \mathrm{~W}$. When the $C_{B}$ was $1000 \mathrm{pF}$, the stored electrical energy was $2.4 \mathrm{~mJ}$, the repetition rate was $130 \mathrm{~Hz}$, and the transfer electrical energy from the boosting capacitor to the load was evaluated to be $0.3 \mathrm{~W}$. It was found that the transfer electrical power in both cases was almost the same. The voltage added to the load was multiplied by four times due to the voltage amplification of flame jet EHD, and the transfer electrical power to the load was amplified by 16 times the original power. Thus, the transferred electrical power was finally increased to $5 \mathrm{~W}$, which was the effect of the voltage amplification of flame jet EHD. The multiplication was remarkably significant.

The maximum power from the DC power supply was $10 \mathrm{~W}$. However, we could not obtain adequate capabilities from the rectification circuit because the load was very small. If we had used load with high levels of current, the energy efficiency of the rectification circuit should have been improved. The three main contributions to improvements were: 1) using fewer variations in capacitance for the boosting capacitor, 2) using a flat capacitor with reduced weight, and 3) using a small flame source. Thus, using these would reduce the weight of the instrument.

\section{Conclusion}

A simple method of producing high voltage pulses based on electrostatic induction without moving parts was introduced. Sawtoothed voltage pulses with short rise times and repetitions of a few $100 \mathrm{~Hz}$ were actually produced in this experiment. The experimental results for generating the voltage pulses were goodly consistent with the numerically calculated ones. We demonstrated that high voltage pulses could be produced by a combination of flame jet induced EHD 
power generation and a boosting capacitor with low consumption of electricity in the load. The power consumption was one order lower than that using normal relaxed self-excited oscillation, and the transferred electrical energy from the boosting capacitor to the load was multiplied to $5 \mathrm{~W}$.

This research did not receive any specific grant from funding agencies in the public, commercial, or not-for-profit sectors.

\section{References}

[1] C. K. Patel, "Continuous-Wave Laser Action on Vibrational-Rotational Transitions of CO2". Physical Review, 136 (5A) (1964) A1187.

[2] M. K. Matzen, "Z Pinches as Intense X-ray Sources for High-energy Density Physics Applications", Physics of Plasmas, 4 (1997) 1519.

[3] H. Akiyama, S. Sakai, T. Sakugaw, and T. Namihira, "Environmental Applications of Repetitive Pulsed Power", IEEE Trans. Dielectr. and Electr. Insulat., 14 (2007) 825.

[4] B. Sun, M. Sato, "Use of a Pulsed High-voltage Discharge for Removal of Organic Compounds in Aqueous Solution", J. Phys. D: Appl. Phys., 32 (1999) 1908.

[5] J. S. Clements, M. Sato, R. H. Davis, "Preliminary Investigation of Prebreakdown Phenomena and Chemical Reactions using a Pulsed High-voltage Discharge in Water", IEEE Trans. Ind. Appl., 23 (1987) 224.

[6] I. V. Lisitsyn, H. Nomiyama, S. Katsuki and H. Akiyama, "Thermal Processes in a Streamer Discharge in Water, "IEEE Trans. Dielectr. Electr. Insulat., 6 (3), (1999) 351.

[7] M. Rezal, Dahaman Ishak, M. Sabri, "High Voltage Magnetic Pulse Generation using Capacitor Discharge Technique", Alexandria Engineering Journal, 53 (2014) 803.

[8] T. Shao, G. S. Sun, P. Yan, S. C. Zhang, "Breakdown Phenomena in Nitrogen due to Repetitive Nanosecond-pulse", IEEE Trans. Dielectr. Electr. Insul., 14 (2007) 813.

[9] E. L. Neau, "Environmental and Industrial Applications of Pulsed Power Systems”, IEEE Trans. Plasma Sci., 22 (1994) 2.
[10] F. Fukawa, N. Shimomura, T. Yano, "Application of Nanosecond Pulse Power to Ozone Production by Streamer Corona", IEEE Trans. Plasma Sci., 36 (2008) 2592.

[11] F. Yan, B. Lin, C. Zhu, Y. Zhou, X. Liu, C. Guo, Q. Zou, "Experimental Investigation on Anthracite Coal Fragmentation by High-voltage Electrical Pulses in the Air Condition: Effect of Breakdown Voltage", Fuel, 183 (2016) 583.

[12] S. Katsuki, H. Akiyama, A. Abou-Ghazala and K. H. Schoenbach, "Parallel Streamer Discharges Between Wire and Plane Electrodis in Water", IEEE Trans. Dielectr. Electr. Inslat., 9 (2002) 498.

[13] M. Sato, T. Tokutake, T. Ohshima and A. T. Sugiarto," Aqueous Phenol Decomposition by Pulsed Discharges on the Water Surface“, IEEE Trans. Ind. Appl., 44, (2008) 1397.

[14] H. Li, A. Lukanin, A. Tskhe, S. Sosnovskiy, "Multifunctional Generator of High-voltage Microsecond Pulses", J. of Electrostatics, 90 (2017) 74

[15] T. Shao, et al., "Excitation of Atmospheric Pressure Uniform Dielectric Barrier Discharge using Repetitive Unipolar Nanosecond-pulse Generator", IEEE Trans. Dielectr. Electr. Insul., 16 (2010) 1830.

[16] T. Shao, D. D. Zhang, Y. Yu, “A Compact Repetitive Unipolar Nanosecond-pulse Generator for Dielectric Barrier Discharge Application”, IEEE Trans. Plasma Sci., 38 (2010) 1651.

[17] K. Yan, E. J. M. van Heesch, A. J. M. Pemen, P. A. H. J. Huijbrechts, F. M. van Gompel, H. van Leuken, and Zdenek Matyá s, "A High-Voltage Pulse Generator for Corona Plasma Generation”, IEEE Trans. Ind. Appl., 38 (3) (2002) 866.

[18] D. Wang, S. Okada, T. Matsumoto, T. Namihira and H. Akiyama, "Pulsed Discharge Induced by Nanosecond Pulsed Power in Atmospheric Air", IEEE Plasma Sci., 38, (2010) 2746.

[19] J.-D. Moon, “A Compact High-voltage Pulse Generator using a Rotary Airhole Sparkgap”, J. of Electrostatics, 65 (2007) 527.

[20] R. A. Ford, Home Made Lightning, McGraw-Hill, New York, 2002.

[21] T. Saiki, "Study on High Voltage Generation Using Flame Column and DC Power Supply", J. of Electrostatics, 70 (2012) 400. 